\section{Evolution of the cost-effectiveness of Endovascular and Open Surgical Repair of Abdominal Aortic Aneurysms}

\author{
Isa C.T. Santos ${ }^{1}$, G. Scott Gazelle ${ }^{2}$, Luís A. Rocha ${ }^{3}$, \\ João Manuel RS Tavares ${ }^{1}$ \\ ${ }^{1}$ Faculdade de Engenharia, Universidade do Porto \\ ${ }^{2}$ Institute for Technology Assessment and Department of \\ Radiology, Massachusetts General Hospital \\ ${ }^{3}$ Institute for Polymers and Composites / I3N, University of \\ Minho
}

\section{Background}

A cost-effectiveness analysis refers to the economic study in which consequences of distinct interventions are evaluated using a single outcome. In healthcare, these analysis measure 'natural' units, such as life-years gained or deaths avoided, and are used to either compare alternative programs with a common health outcome or assess the consequences of expanding an existing program. Commonly, costeffectiveness results are used to support decisions regarding the adoption of medical devices, coverage of treatments or their reimbursement rate [1].

It is commonly accepted that, with time, experience increases as well as success rates and, on the other hand, costs decrease due to economies of scale or the maturation of technologies. However, there is uncertainty regarding the applicability of these assumptions to medical procedures involving medical devices. Here, we study the evolution of the cost-effectiveness of endovascular abdominal aortic aneurysm repair versus the conventional treatment, open surgery. Contrary to what was expected, the effectiveness decreases and costs increase along the time. If this behavior is similar in other procedures, one can question the applicability of cost-effectiveness analysis to support decisions involving medical devices.

\section{Methods}

During October 2011, it was conducted a literature review to identify studies concerning the cost-effectiveness of the treatments of abdominal aortic aneurysms - endovascular aneurysm repair (EVAR) and open surgery. The databases explored were PUBMED, DAHTA, The Cochrane Library, Centre for Reviews and Dissemination and the Cost Effectiveness Analysis Registry; the keywords used were cost-effectiveness, EVAR, open surgery and abdominal aortic aneurysm. Eighteen papers published between 1999 and 2011 were selected for the study.

After a detailed analysis, the following information was extracted: hospital stay, intensive care unit (ICU) stay, operating time and hospitalization costs. The data was plotted and trendlines (polynomial and linear) were defined. It should be noted that, as the information reported in each paper was distinct, some graphs were plotted using few data points.

\section{Results}

The hospital stay increased with time both for the conventional procedure and EVAR, Figure 1. As far as the ICU stay is concerned, it increased for EVAR and decreased for open surgery. The operating time also increased but while the values for open surgery increased moderately in the case of EVAR they almost doubled between 1999 and 2011, Figure 2. The hospitalization costs were converted to US dollars and report to the price year in which the study was conducted, Figure 3.

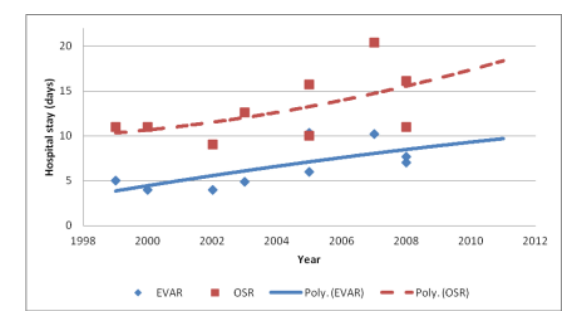

Figure 1: Evolution of the hospital stay

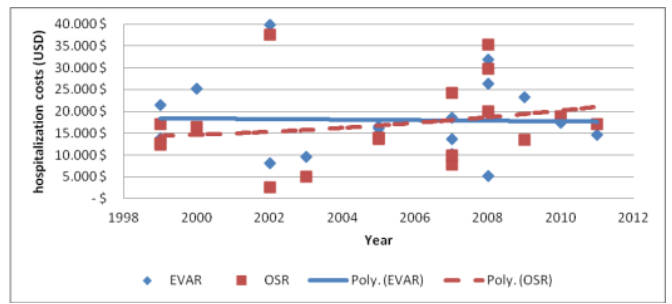

Figure 2: Evolution of the operating time

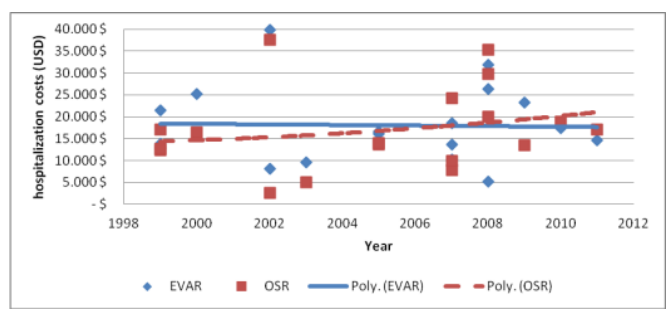

Figure 3: Evolution of the hospitalization costs

\section{Interpretation}

Although this analysis was conducted using few data points, the results point to a decrease in the procedures' efficiency and an increase of costs.

It is possible that the hospital stay, ICU stay and operating time increased because more complicated procedures were being considered. The increase in costs is consistent with the increase of hospital stay, ICU stay and operating time. The increase of the EVAR's costs can be explained by the higher complication rate and the increase of the cost of the stentgraft. However, further studies are needed.

\section{References}

Gold M. R., Siegel J. E., Russell L. B., and Weinstein M. C., eds., 456AD, Cost-Effectiveness analysis in health and medicine, Oxford University Press, USA. 